\title{
Decreased Expression of Angiotensin II Type 1 and Type 2 Receptors in the Brain After Long-Term Administration of Antihypertensive Drugs in Stroke-Prone Spontaneously Hypertensive Rat
}

\author{
Yayoi Nishida ${ }^{1}$, Yasuo Takahashi ${ }^{1}$, Megumi Sugahara-Kobayashi ${ }^{2}$, Kouichi Ishikawa $^{2}$, and Satoshi Asai ${ }^{1, *}$ \\ ${ }^{I}$ Division of Genomic Epidemiology and Clinical Trials, ${ }^{2}$ Department of Pharmacology, \\ Nihon University School of Medicine, 30-1 Oyaguchi-Kamimachi, Itabashi-ku, Tokyo 173-8610, Japan
}

Received December 27, 2007; Accepted February 26, 2008

\begin{abstract}
The present study examined the levels of Angiotensin II type 1 receptor $\left(\mathrm{AT}_{1}\right)$ and type 2 receptor $\left(\mathrm{AT}_{2}\right)$ in the brain stem and cerebral cortex of the stroke-prone spontaneously hypertensive rat (SHR-sp) after long-term treatment with three types of antihypertensive drugs: valsartan, enalapril, and amlodipine. In both tissues, expression of the $\mathrm{AT}_{1}$ was decreased by administration of each drug. Expression of the $\mathrm{AT}_{2}$ was decreased in the cerebral cortex by drug administration, but did not change in the brain stem. This study may contribute to elucidating the relationship between $\mathrm{AT}_{1}$ and $\mathrm{AT}_{2}$ expressions and the effect of antihypertensive drugs in SHRsp brain.
\end{abstract}

Keywords: angiotensin II receptor, antihypertensive drug, brain

Angiotensin II (Ang II) is the principal vasoactive peptide of the renin-angiotensin system, having a variety of physiological actions, including vasoconstriction, aldosterone release, and cell growth (1) Ang II binds two major receptors, Ang II type 1 receptor $\left(\mathrm{AT}_{1}\right)$ and type 2 receptor $\left(\mathrm{AT}_{2}\right)$. The $\mathrm{AT}_{1}$ is mainly expressed in blood vessels, heart, kidney, and adrenal and is also expressed in the brain $(2,3)$. In the brain, the $\mathrm{AT}_{1}$ is also expressed in microvessels (4), and the $\mathrm{AT}_{2}$ is also expressed in neurons (5). The majority of Ang II actions are mediated via the $\mathrm{AT}_{1} ; \mathrm{AT}_{1}$ blockers (ARBs) have been widely used as antihypertensive drugs. The $\mathrm{AT}_{2}$ is reported to be expressed in fetal tissues but is present at low levels in adult tissues and is re-expressed in certain pathological conditions, such as neuronal injury and vascular injury. The $\mathrm{AT}_{2}$ has also been reported to be expressed in the adult rat brain stem, in the inferior olivary nuclei (6) that contain sympathetic premotor neurons for the maintenance of vasomotor tone and blood pressure. Because $\mathrm{AT}_{2}$ activation is believed to oppose $\mathrm{AT}_{1}$-mediated effects (7), it would be of interest to know whether $\mathrm{AT}_{2}$ expression in the brain stem is associated with blood

*Corresponding author. satoshi@med.nihon-u.ac.jp Published online in J-STAGE on April 9, 2008 (in advance) doi: $10.1254 /$ jphs.SC0080027 pressure through an interaction with $\mathrm{AT}_{1}$ signaling. However, $\mathrm{AT}_{1}$ and $\mathrm{AT}_{2}$ expressions and their association in the brain stem are not well defined.

The present study examined the levels of $\mathrm{AT}_{1}$ and $\mathrm{AT}_{2}$ gene expression in the brain stem and cerebral cortex of the stroke-prone spontaneously hypertensive rat (SHR$\mathrm{sp)}$ after long-term treatment with three types of antihypertensive drugs: ARBs, ACE inhibitors, and a calcium channel blocker (CCB); and it clarified the effect of these antihypertensive drugs and blood pressure on $\mathrm{AT}_{1}$ and $\mathrm{AT}_{2}$ expressions. We hope to elucidate the possible role of the $\mathrm{AT}_{1}$ or $\mathrm{AT}_{2}$ in the brain underlying the regulation of the blood pressure.

Forty male SHR-sp/Izm rats were used for this experiment. The experimental protocol was approved by the Ethical Committee of Nihon University, School of Medicine. These animals were allowed standard rat chow (CRF-1; Oriental Yeast, Tokyo) and tap water ad libitum for 1 week. Then they were divided into four groups $(\mathrm{n}=10)$ : 1 , vehicle; 2, valsartan (ARB) (Diovan; Novartis Pharma, Basel, Switzerland) at $30 \mathrm{mg} / \mathrm{kg}$ per day; 3, enalapril maleate salt (ACEI) (Sigma-Aldrich, St. Louis, MO, USA) at $10 \mathrm{mg} / \mathrm{kg}$ per day; and 4, amlodipine (CCB) (Norvasc; Pfizer, New York, NY, USA) at $6 \mathrm{mg} / \mathrm{kg}$ per day. Treatment groups were administered each drug [suspended in carboxy-methyl- 
cellulose (CMC)] orally once a day for 4 weeks from 8 weeks of age. The vehicle group was administered CMC orally once a day for 4 weeks from 8 weeks of age. Systolic blood pressure (SBP) was measured once a week by the tail cuff method, using a Softron BP-98 (Softron, Tokyo). After 4 weeks of treatment (at 12 weeks of age), the brain was dissected into the brain stem and cerebral cortex, which were kept at $-80^{\circ} \mathrm{C}$ until analyzed (8). Figure 1 shows the systolic blood pressure in the treatment groups. SBP increased by about $40 \mathrm{mmHg}$ in the vehicle group during 4 weeks. During 4 weeks, SBP slightly increased in the amlodipine group and slightly decreased in the valsartan and enalapril groups. Although the SBP in the valsartan group and enalapril group was significantly decreased in comparison with that in the amlodipine group from 2 to 4 weeks $(P<0.01)$, SBP in all three treatment groups was significantly decreased in comparison with that in the vehicle group from 1 to 4 weeks $(P<0.01)$.

To validate $\mathrm{AT}_{1}$ and $\mathrm{AT}_{2}$ gene expression, TaqMan RT-PCR assay was performed using an ABI Prism 7700 Sequence Analyzer (PE Applied Biosystems, Foster City, CA, USA) as described previously (9). For the primers and the TaqMan probe for glyceraldehyde-3phosphate dehydrogenase (GAPDH), we used TaqMan

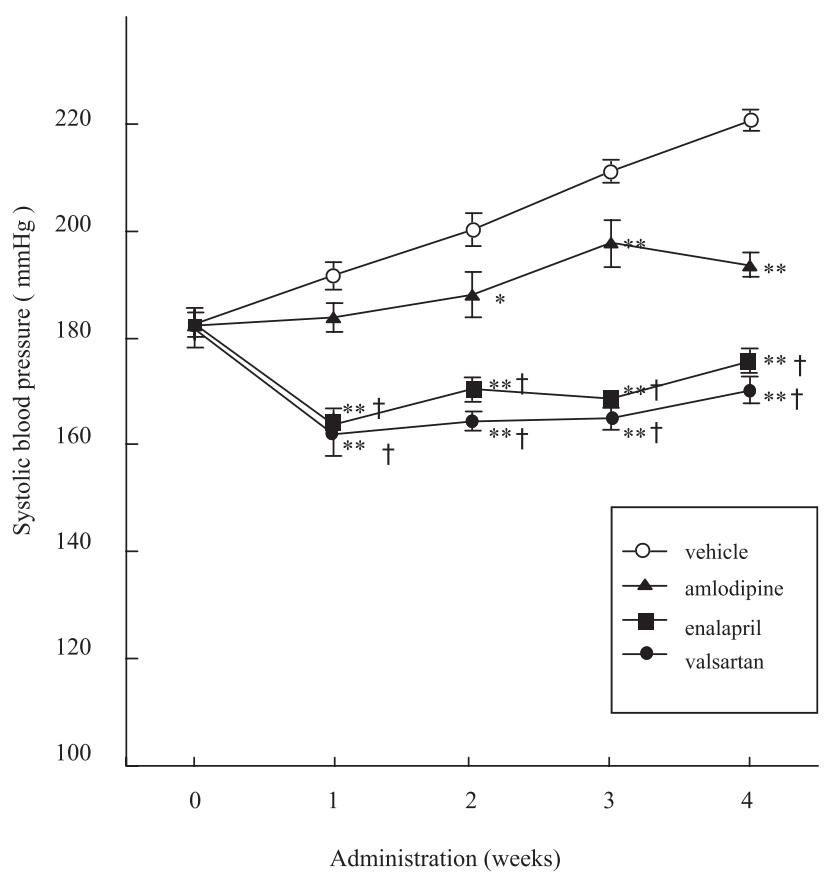

Fig. 1. Effect of antihypertensive drugs on systolic blood pressure in SHR-sp. SHR-sp were administered vehicle (open circle), amlodipine at $6 \mathrm{mg} / \mathrm{kg}$ per day (closed triangle), enalapril at $10 \mathrm{mg}$ $/ \mathrm{kg}$ per day (closed square), or valsartan at $30 \mathrm{mg} / \mathrm{kg}$ per day (closed circle) daily. Data are each a mean \pm S.E.M. $(n=10)$. Significantly different from vehicle, $* P<0.05, * * P<0.01$ and from amlodipine, ${ }^{\dagger} P<0.01$ (Tukey post hoc analysis). rodent GAPDH control reagents (Roche, Basel, Switzerland). Primers and the TaqMan probe for $\mathrm{AT}_{1}$ were as follows $\left(5^{\prime}\right.$ to $\left.3^{\prime}\right)$ : forward primer (TTACCGG CCTTCGGATAAC), reverse primer (ACTCCACCT CAAAACAAGACG), and probe (TGAGCTCATCG GCCAAAAAGCC). Primers and the TaqMan probe for $\mathrm{AT}_{2}$ were as follows ( $5^{\prime}$ to $\left.3^{\prime}\right)$ ): forward primer (CCC TAAAAAGGTGTCCAGCA), reverse primer (TTACA TCTTCAATCTGGCTGTGGCTGA), and probe (AAG GGTTGCCAAAAGGAGTAA). Normalized mRNA levels of the $\mathrm{AT}_{1}$ and $\mathrm{AT}_{2}$ were determined by dividing the levels of each gene by the level of GAPDH. Statistical analysis of TaqMan RT-PCR data was performed with SAS 9.1.3 (SAS Institute, Cary, NC, USA) statistical software (10). One-way analysis of variance (ANOVA) was used to compare $\mathrm{AT}_{1}$ or $\mathrm{AT}_{2}$ expression among treatment groups of valsartan, enalapril, amlodipine, and vehicle, with multiple-comparison
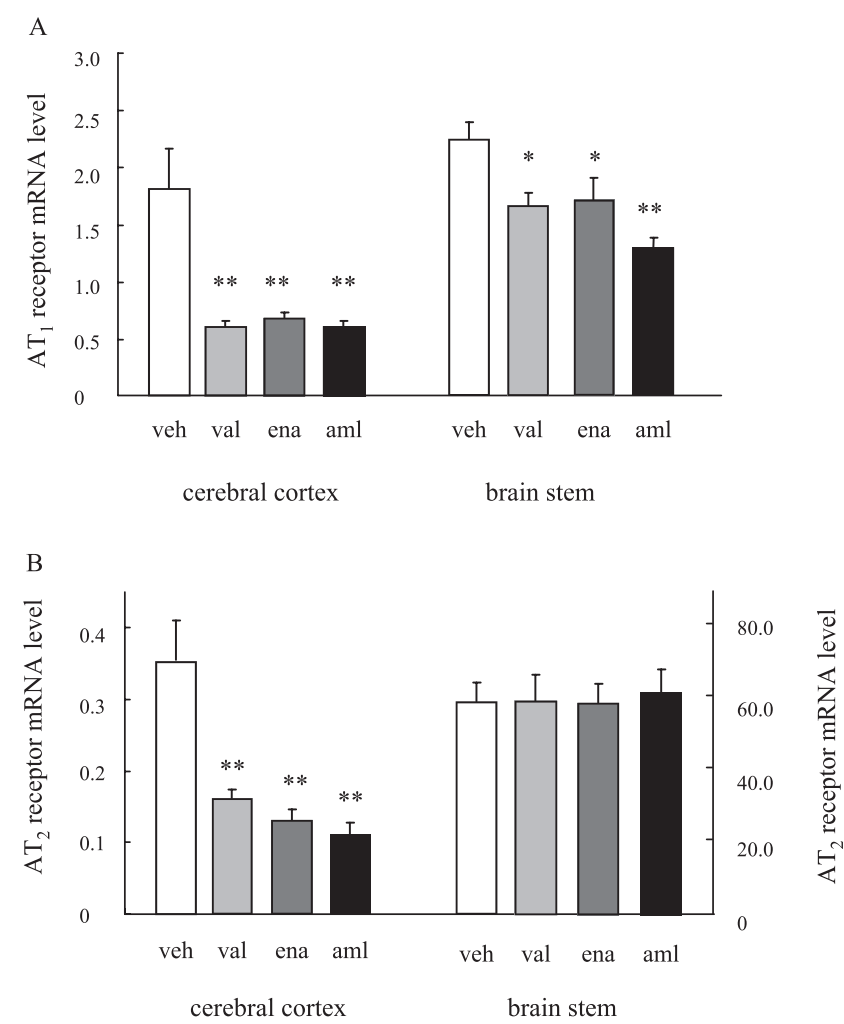

Fig. 2. Effect of antihypertensive drugs on expression of $\mathrm{AT}_{1}$ and $\mathrm{AT}_{2}$ in SHR-sp brain. A: $\mathrm{AT}_{1}$ expression in SHR-sp brain after 4 weeks of administration of antihypertensive drugs (veh: vehicle, val: valsartan, ena: enalapril, aml: amlodipine). Data are each a mean \pm S.E.M. $(\mathrm{n}=10)$. Significantly different from vehicle, ${ }^{*} P<0.05$, $* * P<0.01$ (Tukey post hoc analysis). B: $\mathrm{AT}_{2}$ expression in SHR-sp brain after 4 weeks of administration of antihypertensive drugs. Data are each a mean \pm S.E.M. $(\mathrm{n}=10)$. Significantly different from vehicle, ${ }^{*} P<0.01$ (Tukey post hoc analysis). The left scale shows $\mathrm{AT}_{2}$ expression in the cortex and the right scale shows $\mathrm{AT}_{2}$ expression in the brain stem. 
testing (Tukey-Kramer HSD post hoc analysis) set at a significance level of 0.05 .

Figure 2 shows $\mathrm{AT}_{1}$ and $\mathrm{AT}_{2}$ expression in the brain stem and cerebral cortex of SHR-sp rats treated with valsartan, enalapril, amlodipine, and vehicle. In the cerebral cortex and brain stem, $\mathrm{AT}_{1}$ expression in all three drug treatment groups was significantly decreased in comparison with that of vehicle group $(P<0.05)$, as shown in Fig. 2A. AT 1 expression was not significantly different among the drug treatment groups, despite their different mechanisms of antihypertensive effect. These results suggest that $\mathrm{AT}_{1}$ expression in the brain stem and cerebral cortex was decreased by a blood pressurelowering action of antihypertensive drugs but not by a direct action of antihypertensive agents with different mechanisms of action. Although the blood pressurelowering action of amlodipine was weaker than those of valsartan and enalapril, $\mathrm{AT}_{1}$ expression was tended to be most inhibited by amlodipine. This discrepancy suggests the possibility that the expression of $\mathrm{AT}_{1}$ in the brain stem was influenced not only by a blood pressure lowering action but also by a direct action of amlodipine, such as the blockage of calcium channels and the alteration of nitric oxide production (11). Our results are different from those of the previous studies on the effects of candesartan, enalapril, or amlodipine administration on $\mathrm{AT}_{1}$ expression in the kidney $(12,13)$. The difference may mainly come from the difference in tissues examined, brain or kidney, between the studies, although differences in age and strain of rats used would also be possible causes of the difference. As the blood brain barrier obstructed the penetration of these agents into the brain, it is considered that expression of the $\mathrm{AT}_{1}$ decreased through a reduction of blood pressure by administration of antihypertensive agents. Our present study suggested that expression of the $\mathrm{AT}_{1}$, which mediates the majority of Ang II actions in the brain stem and cerebral cortex, may be influenced by the change of blood pressure, at least in SHR-sp.

The antihypertensive agents affected expression of the $\mathrm{AT}_{2}$ in the cerebral cortex and brain stem in different manners. In the cerebral cortex, $\mathrm{AT}_{2}$ expression in all three drug treatment groups was significantly decreased in comparison with that in the vehicle group $(P<0.05)$, as shown in Fig. 2B. Similar to the case of $\mathrm{AT}_{1}$ expression in the cerebral cortex, this result suggests that expression of the $\mathrm{AT}_{2}$ in the cerebral cortex is decreased by the reduction of blood pressure but not by a direct action of antihypertensive agents. In the brain stem, the difference in $\mathrm{AT}_{2}$ expression was not significant not only between each of the drug treatment groups and the vehicle group, but also among drug treatment groups, as shown in Fig. 2B. These findings suggest that $\mathrm{AT}_{2}$ expression in the brain stem is not directly affected by antihypertensive drugs and is not affected by blood pressure. Therefore, regulation of $\mathrm{AT}_{2}$ expression may be unrelated to blood pressure control and independent of the $\mathrm{AT}_{1}$ signaling system, suggesting an important role of the $\mathrm{AT}_{2}$ in the brain stem. Supporting this possibility, evidence showing effects of $\mathrm{AT}_{2}$ signaling in cerebral protection and neural differentiation has accumulated $(14,15)$. Although expression of the $\mathrm{AT}_{2}$ in the adult brain is limited, these receptors are reported to be upregulated in the brain after ischemic damage, suggesting an important role of $\mathrm{AT}_{2}$ activation in neural protection. In this study, we found that $\mathrm{AT}_{2}$ expression was significantly increased in the brain stem in comparison with that in the cerebral cortex, as shown in Fig. 2. Our findings, in combination with previous reports (15), suggest that the brain stem is more resistant to brain damage including ischemic injury than the cerebral cortex because $\mathrm{AT}_{2}$-mediated neuroprotective activity is stronger in the former than in the latter.

In situ hybridization was not carried out in the present study because mRNA expression of the $\mathrm{AT}_{1}$ and $\mathrm{AT}_{2}$ was low and hard to detect. In the brain, however, it has been reported that these receptors are expressed in microvessels (4) and neurons (5). Therefore, it is important to determine the expression site of $\mathrm{AT}_{1}$ and $\mathrm{AT}_{2}$, that is, neurons or microvessels; and we would like to clarify this next using a microdissection method.

The present study demonstrated that antihypertensive agents decreased expression of the $\mathrm{AT}_{1}$ in the brain stem and cerebral cortex of SHR-sp. In addition, we found that expression of the $\mathrm{AT}_{2}$ was markedly higher in the brain stem than in the cerebral cortex and that antihypertensive agents themselves and reduction of blood pressure did not affect expression of the $\mathrm{AT}_{2}$ in the brain stem. This study may contribute to elucidating the relationship between $\mathrm{AT}_{1}$ and $\mathrm{AT}_{2}$ expressions and the effect of antihypertensive drugs in SHR-sp brain.

\section{Acknowledgments}

This experiment was supported by a Grant-in Aid from the Japanese Ministry of Education, Science, Sports, and Culture to promote advanced scientific research, awarded to Nihon University, and Nihon University Research Grant for Assistant and Young Researcher (07-032)

\section{References}

1 de Gasparo M, Catt KJ, Inagami T, Wright JW, Unger T. International union of pharmacology. XXIII The angiotensin II receptors. Pharmacol Rev. 2000;52:415-472. 
2 Siragy HM. The role of the AT2 receptor in hypertension. Am J Hypertens. 2000;13:62S-67S.

3 von Bohlen und Halbach O, Albrecht D. The CNS reninangiotensin system. Cell Tissue Res. 2006;326:599-616.

4 Ando H, Jezova M, Zhou J, Saavedra JM. Angiotensin II AT1 receptor blockade decreases brain artery inflammation in a stress-prone rat strain. Ann N Y Acad Sci. 2004;1018:345-350.

5 McKinley MJ, McAllen RM, Pennington GL, Smardencas A, Weisinger RS, Oldfield BJ. Physiological actions of angiotensin II mediated by AT1 and AT2 receptors in the brain. Clin Exp Pharmacol Physiol Suppl. 1996;3:S99-S104.

6 Jöhren O, Inagami T, Saavedra JM. Localization of AT2 angiotensin II receptor gene expression in rat brain by in situ hybridization histochemistry. Brain Res Mol Brain Res. 1996;37:192-200.

7 Widdop RE, Jones ES, Hannan RE, Gaspari TA. Angiotensin $\mathrm{AT}_{2}$ receptors: cardiovascular hope or hype. $\mathrm{Br} \mathrm{J}$ Pharmacol. 2003;140:809-824.

8 Nishida Y, Nagata T, Takahashi Y, Sugahara-Kobayashi M, Murata A, Asai S. Alteration of serum/glucocorticoid regulated kinase-1 (sgk-1) gene expression in rat hippocampus after transient global ischemia. Brain Res Mol Brain Res. 2004; 123:121-125.

9 Takahashi Y, Ishii Y, Nishida Y, Ikarashi M, Nagata T, Nakamura $\mathrm{T}$, et al. Detection of aberrations of ubiquitin-conjugating enzyme E2C gene (UBE2C) in advanced colon cancer with liver metastases by DNA microarray and two-color FISH. Cancer Genet Cytogenet. 2006;168:30-35.
10 Takahashi Y, Masuda H, Ishii Y, Nishida Y, Kobayashi M, Asai $\mathrm{S}$. Decreased expression of thioredoxin interacting protein mRNA in inflamed colonic mucosa in patients with ulcerative colitis. Oncol Rep. 2007;18:531-535.

11 Zhang X, Hintze TH. Amlodipine releases nitric oxide from canine coronary microvessels: an unexpected mechanism of action of a calcium channel-blocking agent. Circulation. 1998;97:576-580.

12 Inada Y, Wada T, Ojima M, Sanada T, Shibouta Y, Kanagawa $\mathrm{R}$, et al. Protective effects of candesartan cilexetil (TCV-116) against stroke, kidney dysfunction and cardiac hypertrophy in stroke-prone spontaneously hypertensive rats. Clin Exp Hypertens. 1997;19:1079-1099.

13 Kishida M, Otsuka F, Ogura T, Kataoka H, Nakamura Y, Yamauchi T, et al. Chronic treatment with amlodipine modulates adrenocortical angiotensin II receptors in spontaneously hypertensive rats. Life Sci. 2001;69:113-122.

14 Li JM, Mogi M, Tsukuda K, Tomochika H, Iwanami J, Min LJ, et al. Angiotensin II-induced neural differentiation via angiotensin II type 2 (AT2) receptor-MMS2 cascade involving interaction between AT2 receptor-interacting protein and Src homology 2 domain-containing protein-tyrosine phosphatase 1 . Mol Endocrinol. 2007;21:499-511.

15 Mogi M, Li JM, Iwanami J, Min LJ, Tsukuda K, Iwai M, et al. Angiotensin II type-2 receptor stimulation prevents neural damage by transcriptional activation of methyl methanesulfonate sensitive 2. Hypertension. 2006;48:141-148. 